\title{
La notion de transfert appliquée au français comme langue étrangère
}

\author{
Hans Petter Helland (Université d'Oslo, Norvège)
}

\begin{abstract}
Our main objective in this paper is to show how linguistic properties can be transferred from one system to another. Consequences of transfer are evaluated, both positive and negative, for the acquisition of possessives in French L2 or L3 by Norwegian learners. We take as a starting point a comparative description of the possessive systems in French and Norwegian and examine interlanguage grammars of adult learners of French both in a Norwegian university setting and in an immersion context. On the basis of results from production-comprehension tests we can predict and explain interlanguage errors in the students' L2-grammar (negative transfer) using comparative grammatical constraints (reflexive-irreflexive contrasts, binding constraints, explicit marking of the possessor, orientation towards the possessor or the possessum, etc.). We can also predict cases where the learners make fewer errors or no errors at all (positive transfer). In this way we are able to measure the importance of transfer, both negative and positive, for the acquisition of French as foreign language by Norwegian adult learners.
\end{abstract}

Keywords: French as a foreign language, L2, French-Norwegian, possessives, transfer

\section{Introduction}

Dans cette contribution, notre objectif est de montrer comment des propriétés linguistiques peuvent être transférées d'un système linguistique à un autre. On distingue généralement trois types de transfert : transfert de L1 à L2, transfert de L2 à L1 et transfert de L2 à L3. C'est le premier type qui nous intéresse principalement dans ce travail. Notre objectif est d'évaluer les conséquences du transfert, à la fois positif et négatif, pour l'acquisition des possessifs en français comme langue étrangère par des apprenants norvégiens. Nous examinons la grammaire de l'interlangue d'étudiants norvégiens à la fois dans un contexte étranger (tests effectués à l'Université d'Oslo avec des étudiants norvégiens du français langue étrangère) et dans un contexte d'immersion (tests effectués à l'Université de Caen, France avec des étudiants norvégiens qui passent une année à l'étranger pour étudier le français comme langue étrangère). Les tests portent sur la production-compréhension (tests de traduction). Sur la base des résultats obtenus, nous pouvons prévoir et expliquer les erreurs (transfert négatif) dans les interlangues des apprenants en tenant compte de contraintes grammaticales comparatives (formes réfléchies, non réfléchies, contraintes de liage, marquage explicite du possesseur, orientation vers le possesseur ou le possédé, etc.). Nous pouvons également prévoir des cas où les apprenants feront moins d'erreurs (transfert positif). Il sera donc possible de mesurer l'importance du transfert, à la fois négatif et positif, pour l'acquisition du français comme langue étrangère par des apprenants norvégiens. 
Nous illustrerons nos points théoriques sur la base d'une description comparative entre les systèmes possessifs en français et en norvégien que nous avons entreprise dans Helland $(2017,2018)$. Les deux langues ont des formes possessives à la troisième personne en $s^{*}:$ son / sa / ses pour le français et sin / si / sitt /sine pour le norvégien. Cependant, seul le norvégien opère l'opposition entre des formes réfléchies en $s i{ }^{*}$ et des formes non réfléchies : hans / hennes / dens / dets / deres. On constate ainsi que des formes morphologiquement et syntaxiquement apparentées en $s$ - ont des propriétés fort différentes dans les deux langues en question.

Telle sera notre démarche : nous introduirons d'abord la notion de transfert en L2 (ou en L3) avant de l'appliquer, en termes abstraits, à l'acquisition du français comme langue étrangère. Ensuite, nous donnerons des exemples concrets du transfert linguistique dans le domaine des possessifs. Les données sont tirées de notre corpus d'apprenants universitaires norvégiens.

\section{Le transfert linquistique}

Très souvent dans la littérature consacrée à l'acquisition des langues étrangères, la notion de transfert est associée à la linguistique contrastive des années 1950 (Odlin, Yu 2016). Comme le montrent ces auteurs, le phénomène de transfert, comme le terme même, est cependant connu bien avant les années 1950 et l'analyse contrastive. Aujourd'hui, on remplace en général le terme de transfert par celui, plus neutre, de cross-linguistic influence (influence trans-linguistique) (Jarvis, Pavlenko 2008, et bien d'autres). Néanmoins, comme les deux termes continuent à coexister, autant les considérer comme synonymes.

Le transfert se définit comme l'influence de connaissances linguistiques que possède le locuteur dans une langue $x$ sur des connaissances linguistiques que le même locuteur a (ou acquiert) dans une autre langue $y$. Le plus souvent, il s'agit de transfert entre la langue maternelle du locuteur (L1) et une deuxième langue (L2) qu'il est en train d'apprendre. Mais il peut également être question de transfert dans le sens inverse L2 $\rightarrow$ L1. Quand on parle de similarités ou de différences entre la L1 et la L2, la L2 est généralement considérée comme toute langue qui s'acquiert après la langue maternelle. Ces dernières années, il y a cependant un intérêt croissant pour distinguer l'acquisition d'une deuxième langue (L2) et d'une troisième langue (L3). Le transfert pourra ainsi s'établir, non seulement entre la L1 et la L2, mais également entre la L1 et la L3 et la L2 et la L3, éventuellement dans le sens inverse. Et comme nous le verrons par la suite, on ne s'arrête pas là. Il y a encore d'autres relations de transfert possibles, ce qui fait des relations trans-linguistiques un domaine acquisitionnel fort complexe. En grammaire générative, le terme de transfert s'emploie également pour distinguer le rapport entre les connaissances innées (grammaire universelle GU) et la langue apprise. La GU est un système abstrait de connaissances grammaticales reflétant des structures communes à toutes les langues, dont l'enfant dispose avant d'apprendre sa langue maternelle ou une langue étrangère. Il est généralement admis dans ce genre de théories, que la GU joue un rôle crucial, du moins dans l'acquisition de la L1, mais même pour l'acquisition d'une langue étrangère (L2, L3...), on a essayé de justifier la contribution (variable) de la GU dans le processus acquisitionnel (Benati \& Angelskova 2016 ; Eide et al. 2015). A partir d'un tel point de vue théorique, le transfert peut s'établir, bidirectionnellement, non seulement entre les langues stockées dans le cerveau du locuteur (L1, L2, L3...), mais également entre la grammaire universelle et les langues acquises (L1, L2, 
L3...). Pour étayer notre argumentation dans ce travail, nous limiterons notre discussion essentiellement au transfert entre la L1 et la L2.

\subsection{Le transfert entre L1 et L2}

La relation de transfert de loin la plus importante est celle qui relie la langue maternelle à la nouvelle langue qui s'apprend. Pour des raisons de simplicité - et conformément à la tradition linguistique - nous parlerons, sauf indication contraire, de la L2 tout court. D'un point de vue historique, on a affirmé que le transfert relève essentiellement du domaine phonétique/phonologique et lexical (Odlin, 2003, 439-441 ; Jarvis \& Pavlenko 2008). Pour la relation entre le norvégien comme L1 et le français comme L2, on trouvera facilement des exemples de ce type. En norvégien, la consonne fricative $s$ à la graphie se prononce $[s]$, sans voisement, entre deux voyelles graphiques (et prononcées), comme dans les noms, rose, Lise, etc. Et ce contrairement à la fricative sonore du français [z] dans les mêmes contextes (suivie d'un $e$ muet). On s'attendra alors à ce que les apprenants norvégiens du français comme langue étrangère fassent des erreurs dans leur interlangue du français comme L2 optant pour la consonne non sonore là où le français exige la consonne sonore. De tels effets sont largement attestés dans les cours du français comme langue étrangère dans les établissements scolaires en Norvège. Dans le domaine lexical, les faux amis sont également bien connus. Le mot farin en norvégien signifie sucre alors que le correspondant lexical du français farine a une signification très distincte (= grains de blé, voir Lorentzen 2010). Il en va de même pour un grand nombre de paires de mots, telles que professor $(\mathrm{N})$ - professeur (F) ; konduktфr (N) - conducteur (F), qui n'ont pas la même signification dans les deux langues. Des erreurs dans l'interlangue des apprenants sont dès lors prévisibles. Or, le transfert ne se limite pas à des effets de prononciation ou de signification lexicale. En effet, comme nous le verrons par la suite, le transfert peut toucher à tous les domaines des connaissances linguistiques, y compris la morphologie et la syntaxe (Jarvis 2017, Odlin \& Yu 2016). Si le transfert induit à des erreurs dans la production linguistique L2 de l'apprenant, on parlera de transfert négatif (Odlin \& Yu 2016). Si par contre les similarités entre la L1 et L2 facilitent l'apprentissage, on parlera de transfert positif.

\section{Les systèmes possessifs en français et en norvégien}

Le fonctionnement des possessifs est bien plus complexe en norvégien qu'en français. Certes, le français a des formes spécifiques pour l'emploi adjectival des possessifs (le mien, le tien, le sien, etc.), mais quand le possessif se comporte comme un déterminant, il se place, sans exception, devant le nom possédé tout en variant en personne grammaticale avec le possesseur et en genre (au singulier) ou en nombre (singulier et pluriel) avec le possédé (Fabricius Hansen et al. 2017, Helland 2017, 2018) :

(1) Mon fils / ma table / mes affaires, etc.

Contrairement au français, le norvégien distingue trois genres au singulier (le masculin, le féminin ou le neutre), mais il établit en même temps une distinction 
morphologique à la troisième personne (du singulier et du pluriel) entre formes réfléchies et non réfléchies ${ }^{1}$ :

(2) Sin bil / hans bil / hennes bil, etc.

$\mathrm{Sa}$ :REFL voiture (à lui ou à elle) / sa :IRREFL voiture (à lui) / sa :IRREFL voiture (à elle), etc.

Cela dit, la forme potentiellement ambiguë entre l'interprétation réfléchie ou non réfléchie du français dans (3) peut correspondre à (4) ou à (5) du norvégien où l'opposition entre le réfléchi ou le non réfléchi est explicite ${ }^{2}$ :

(3) Pierre $_{i}$ a retrouvé $\mathrm{sa}_{\mathrm{i} / \mathrm{j}}$ voiture.

(4) Peter ${ }_{\mathrm{i}}$ fant igjen $\sin _{\mathrm{i}}$ bil.

Pierre a retrouvé sa:REFL voiture.

(5) Peter $_{i}$ fant igjen hans ${ }_{j}$ bil.

Pierre a retrouvé sa:IRREFL sa voiture.

Les formes non réfléchies du norvégien (6) - tout en étant invariables en fonction du nom possédé - se rapportent au possesseur pour le genre (hans $(m, s g)$, hennes $(f, s g)$ ) ou le trait non humain (dens ( $m$ et $f$, $s g$ ), dets (n(eutre), $s g)$ ) du singulier. Au pluriel, deres est la seule forme disponible. Les formes réfléchies par contre (7)-(8) ont la même forme au singulier et au pluriel, mais elles varient en genre et nombre selon le nom possédé :

(6) Peter fant igjen hans $\mathrm{b}_{\mathrm{j}}$ bil / hennes $\mathrm{s}_{\mathrm{j}} \mathrm{bil}_{\mathrm{l}} /$ deres $_{\mathrm{j}}$ bil / dens $_{\mathrm{j}}$ leke / dets $_{\mathrm{j}}$ leke.

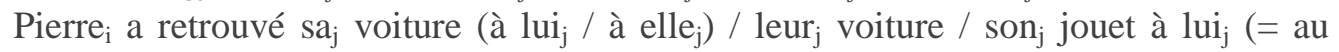
chien $\left._{\mathrm{j}}\right) /$ son $_{\mathrm{j}}$ jouet à lui $_{\mathrm{j}}\left(=\right.$ à l'animal $\left.{ }_{\mathrm{j}}\right)$.

(7) Peter $_{\mathrm{i}}$ fant igjen $\sin _{\mathrm{i}}$ bil.

Pierre ${ }_{i}$ a retrouvé $\mathrm{sa}_{\mathrm{i}}$ voiture.

(8) $[\text { Peter og Marie }]_{\mathrm{i}}$ fant igjen $\sin _{\mathrm{i}}$ bil.

Pierre et Marie ont retrouvé leur voiture.

De plus - élément de complexité supplémentaire - les possessifs du norvégien peuvent se placer devant ou après le nom possédé (9)-(10). Antéposés, ils se comportent grosso modo comme les possessifs du français. Postposés, le possessif exige que le nom soit défini, d'où le critère de double définitude (Fabricius Hansen et al. 2017) :

(9) Min bil, sin bil, hans bil, etc.

Ma voiture, sa:REFL voiture, sa:IRREFL voiture, etc.

(10) Bil-en min, bil-en sin, bil-en hans, etc.

Ma voiture, sa:REFL voiture, sa :IRREFL voiture, etc.

\footnotetext{
${ }^{1}$ Dans la traduction des exemples norvégiens, nous indiquerons par REFL ou IRREFL s'il s'agit d'une forme réfléchie ou non réefléchie (ou irréfléchie).

${ }^{2} \mathrm{La}$ co-indexation entre le groupe nominal sujet et le possessif marque l'interprétation réfléchie (et coréférentielle) entre les deux expressions nominales. Si les expressions nominales portent des indices distincts, elles réfèrent à des personnes distinctes.
} 
Sur la base de telles précisions, nous aboutissons à un certain nombre de correspondances entre les possessifs du français et du norvégien (Fabricius Hansen et al. 2017, Helland 2017, Helland à paraître) :

- Le français et le norvégien possèdent des séries de formes qui marquent la relation entre un possesseur et un possédé.

- Le marquage morphologique en fonction du possesseur ou du possédé n'est pas le même dans les deux langues :

○ Le norvégien a trois genres (masculin, féminin, neutre) contrairement aux deux genres (masculin, féminin) du français ;

- Seul le norvégien fait la distinction (à la troisième personne du singulier et $\mathrm{du}$ pluriel) entre formes réfléchies et formes non réfléchies. Les formes non réfléchies se rapportent au possesseur tout en restant invariables par rapport au nom possédé. Les formes réfléchies dépendent de l'interprétation référentielle du groupe nominal sujet. Les formes réfléchies en $s^{*}$ sont identiques à la troisième personne du singulier et du pluriel. Dans les deux cas, les possessifs s'accordent avec le nom possédé ;

- Le français n'a pas de formes proprement réfléchies. Cela implique qu'à la troisième personne du singulier, les formes en $s^{*}$ du français (son, sa, ses, etc.) sont ambiguës entre un emploi réfléchi et non réfléchi. A la troisième personne du pluriel, le français a des formes spécifiques en leur*. Autrement dit, une forme réfléchie en $s^{*}$ du norvégien peut se rapporter à un possesseur singulier ou pluriel alors que les formes en $s^{*}$ du français, non spécifiées pour l'opposition réfléchie-non réfléchie, se limitent à la troisième personne du singulier.

- En français, les déterminants possessifs se placent, sans exception, devant le nom tête du groupe nominal, position également valable en norvégien. Mais pour cette langue, le cas non marqué reste cependant la postposition du possessif combinée avec le double marquage de définitude (suffixe nominal de définitude suivi du possessif).

Dans la section suivante, nous allons introduire des tests linguistiques pour examiner des effets de transfert entre les deux systèmes possessifs.

\section{Tests linguistiques}

Il s'agit de deux tests de traduction qui ont été effectués à l'Université d'Oslo et à l'Université de Caen entre 2015 et $2016 .{ }^{3}$ Les groupes suivaient une formation de français langue étrangère dans le système universitaire norvégien, d'une part dans un contexte étranger (Université d'Oslo), d'autre part dans un contexte d'immersion (Université de Caen). Les tests contenaient deux textes de traduction, un texte norvégien à rendre en français, avec 21

\footnotetext{
${ }^{3}$ Ce travail d'ordre théorique et empirique est le fruit d'une collaboration entre Bergljot Behrens, Cathrine Fabricius-Hansen, Anneliese Pitz et Hans Petter Helland dans le cadre du projet SPROSS issu de l'Université d'Oslo.
} 
informateurs à Oslo et 14 étudiants à Caen. Ensuite, un deuxième texte à traduire du français en norvégien, avec 33 informateurs à Oslo et 13 à Caen. Chaque groupe suivait des cours de français au niveau universitaire depuis environ deux mois.

Les étudiants n'étaient pas au courant du fait qu'on les testait pour des possessifs. Les textes contenaient cependant un nombre relativement important de possessifs qu'il fallait traduire. Ci-dessous nous présenterons les deux textes en mettant en gras - pour des raisons de lisibilité - les expressions possessives :

\section{Traduisez le texte suivant en français :}

Edvard Grieg var en norsk komponist. I Norge er han best kjent for sin musikk til diktene av Aasmund Olavsson Vinje, mens det i utlandet var musikken hans til Henrik Ibsens tekster som ble lagt mest merke til. Grieg tilbrakte mye tid i utlandet, og var ofte på konsertreiser, hvor han akkompagnerte sin kone Nina Hagerup. Hun opptrådte også ofte sammen med mannen sin, og deres konserter i Europa fikk gode kritikker. Hun var en utmerket pianist, men det var først og fremst stemmen hennes og dens helt spesielle klang som fascinerte Edvard Grieg. Hagerup fortsatte også å delta på konserter etter sin manns død.

Grieg fikk sin første musikkutdannelse av moren sin. Han dro allerede som 15-åring til musikkhøyskolen i Leipzig for å studere, men fikk etter hvert et anstrengt forhold til skolen og lærerne der på grunn av deres innstilling til musikken hans. I 1867 giftet han seg med Nina Hagerup, som faktisk var kusinen hans. Deres eneste barn døde bare 13 måneder gammel, noe som var spesielt tungt for Nina, som også hadde mistet foreldrene sine. Moren hennes var teaterinstruktør og hadde hatt stor betydning for henne. I 1884 flyttet Grieg og Hagerup til Troldhaugen, hvor de bodde resten av livet.

I dag er Troldhaugen museum, og huset med møblene er godt bevart. Her kan man lære mye interessant om Edvard Grieg, hans kone og deres liv i utkanten av Bergen. På Troldhaugen blir det også arrangert konserter med band både fra Norge og fra utlandet, som kommer til Bergen med sine tolkninger av Griegs musikk.

\section{Traduisez le texte suivant en norvégien :}

\section{Nicolas Sarkozy et Carla Bruni}

Il est bien rare que Nicolas Sarkozy manque un concert de sa belle Carla Bruni-Sarkozy, qui avait dû mettre entre parenthèses sa carrière lors de son passage à l'Élysée. Il est sans doute son plus grand fan. Et lorsqu'il arrive devant la salle, on est souvent en droit de se demander si les personnes présentes ne viennent pas assister à un meeting de leur ancien président.

Depuis le début de sa tournée pleine de poésie en novembre dernier, Carla Bruni a pu compter sur le soutien inconditionnel de son époux, qui a assisté à plus d'une vingtaine de ses concerts. À Béziers, le 17 janvier dernier, il avait dit : "C'est mon dix-septième concert, mon dix-septième!

Ce qui ne change pas, c'est l'ovation que reçoit Nicolas Sarkozy par le public, et chaque concert est donc l'occasion pour lui de constater que sa popularité reste intacte. Dès qu'il le peut, il met Carla en avant, ses talents de chanteuse, son aisance sur scène. Lorsque dans la coulisse Nicolas Sarkozy se montre un peu trop élogieux, son épouse n'hésite pas 
à le reprendre : "Ne parle pas trop, mon amour, ça risquerait de se retrouver dans la presse." Et lui de répondre : "Vous voyez, c'est elle la patronne de notre couple !"

Si c'est elle la patronne de leur couple, Nicolas Sarkozy reste pour beaucoup le patron de l'UMP et notre candidat le plus crédible à l'élection présidentielle de 2017. Mais si la politique fait encore partie intégrante de sa vie, Nicolas Sarkozy n'oublie pas sa famille. La preuve avec cet avion privé que loue l'ancien président pour certains concerts en province, afin de permettre à son couple de retrouver rapidement leurs enfants.

\author{
soutien (m) - støtte \\ inconditionnel - betingelsesløs \\ ovation (f) - hyllest \\ aisance (f) - letthet \\ élogieux - rosende
}

\author{
louer - å leie \\ patron (m), patronne (f) - sjef \\ crédible - troverdig \\ preuve (f) - bevis \\ en province $-i$ provinsen
}

Tournons-nous vers des exemples concrets tirés des tests de traduction.

\section{Effets de transfert}

\subsection{Traduction du norvégien en français}

Nous examinerons des cas qui nous permettent de décider s'il y a des effets de transfert entre le norvégien comme L1 et le français comme L2 (éventuellement L3). Rappelons que le système des possessifs en norvégien est morphologiquement et syntaxiquement plus complexe que celui du français. Contrairement au français, le norvégien oppose, à la troisième personne, des formes réfléchies à des formes non réfléchies. Les deux langues ont cependant des formes en $s^{*}$. En français, il s'agit de formes, exclusivement réservées à la troisième personne du singulier, - non marquées pour l'opposition réfléchi-non réfléchi, le genre ou le sens humain-non humain. En norvégien, par contre, les formes en $s^{*}$ sont des formes réfléchies - masculines ou féminines - de la troisième personne, à la fois du singulier et du pluriel, et opposées à des formes non réfléchies spécifiques. L'apprenant norvégien $\mathrm{du}$ français comme L2 part donc d'un système morphologiquement et syntaxiquement très complexe de sa langue maternelle pour acquérir un système moins complexe.

On s'attend alors à ce qu'il y ait peu d'erreurs dans la production linguistique de l'apprenant norvégien là où les conditions d'emploi des formes en $s^{*}$ se chevauchent $\left(s^{*} \rightarrow\right.$ $\left.s^{*}\right)$. Une telle hypothèse est largement confirmée par nos données. En voici deux exemples tirés de la traduction du norvégien en français :

(11) Hagerup fortsatte også å delta på konserter etter sin manns død. Hagerup a continué à participer à des concerts après la mort de son:REFL mari.

(12) ... (noe som var spesielt tungt for Nina), som også hadde mistet forledrene sine. ... (ce qui était particulièrement dur pour Nina), qui avait également perdu ses:REFL parents.

Les déterminants possessifs réfléchis de (11) et de (12) correspondent à des formes en $s^{*}$ du français, et il n'y a pratiquement pas d'erreurs dans les traductions. Malgré quelques 
hésitations d'ordre lexical concernant le choix du nom mari (11), qu'il s'agisse du groupe d'Oslo ou de Caen, on atteste cent pour cent de réussite pour la traduction du possessif (son mari). Il en va de même pour le possessif de (12) (ses parents). Sauf une seule erreur (*sa parents) liée à l'accord du possessif avec le nom possédé dans le groupe de Caen et une seule erreur dans le groupe d'Oslo (*sons parents) - les informateurs établissent parfaitement la correspondance entre les formes en $s^{*}$ dans les deux langues. On parlera alors de transfert positif entre le norvégien et le français.

Même pour les formes non réfléchies du norvégien à la troisième personne du singulier, pour lesquelles $s^{*}$ reste la seule option en français, les résultats sont relativement clairs : musikken hans (sa:IRREFL musique à lui). Que ce soit à Oslo ou à Caen, la plupart des informateurs, malgré quelques erreurs concernant le genre du nom possédé (*son musique), choisissent des formes en $s^{*}$ dans leurs traductions françaises : 16/21 dans le groupe d'Oslo et 13/14 à Caen.

Par contre, si les conditions d'emploi des formes en $s^{*}$ ne sont pas les mêmes dans les deux langues, on s'attend à un taux plus élevé d'erreurs. Le français opère une opposition entre un déterminant possessif à la troisième personne du singulier et du pluriel, et ce en fonction du nombre du possesseur : $s^{*}$ - leur*. Pour les possessifs réfléchis du norvégien, les formes en s* d'un possesseur singulier ou pluriel sont cependant les mêmes. Dans un exemple comme (13), la forme en $s^{*}$ du norvégien ne peut être rendue en français par $s^{*}$ :

(13) På Troldhaugen blir det også arrangert konserter med band både fra Norge og fra utlandet, som kommer til Bergen med sine tolkninger av Griegs musikk.

(A Troldhaugen, on organisera également des concerts avec des orchestres venant aussi bien de la Norvège que de l'étranger), qui viennent à Bergen avec leurs:REFL interprétations de la musique de Grieg.

Malgré ce fait, sur les 11 informateurs dans le groupe de Caen qui ont répondu à cette question, 6 choisissent une variante en $s^{*}$. Parallèlement, dans le groupe d'Oslo, 3/8 utilisent une forme en $\mathrm{s}^{*}$ (ses interprétations). Ceci est un exemple frappant de transfert négatif.

Il y a donc une tendance chez les informateurs norvégiens à (sur-) généraliser l'emploi des formes en $s^{*}$. Même avec le sens pluriel du possessif non réfléchi en norvégien (deres konserter $i$ Europa - leurs:IRREFL concerts en Europe), qui correspond à une forme particulière en français, il y a des occurrences en $s^{*}$ dans les traductions : 4/20 dans le groupe d'Oslo, mais seulement 1/14 dans le groupe de Caen. (14) :

Un autre contexte frappant pour lequel on trouve bien des erreurs en $s^{*}$ est celui de

(14) (I 1884 flyttet Grieg og Hagerup til Troldhaugen), hvor de bodde resten av livet. (En 1884, Grieg et Hagerup ont déménagé à Troldhaugen), où ils habitèrent pour le reste de la vie:DEF.)

Il est bien connu (Woldsnes 2013) que le marquage de la relation possessive est moins prononcé en norvégien qu'en français. Ainsi, le groupe nominal défini livet (= la vie) du norvégien (qui contient le suffixe défini -et) tend à être rendu en français par un possessif. Le groupe d'Oslo a bien saisi cette différence de marquage dans les deux langues optant souvent pour un possessif. Cependant, sur les 10 réponses, pour cette phrase contenant un possessif, 5/11 choisissent la forme erronée en $s^{*}$, nouvelle occurrence de transfert négatif. Dans le groupe de Caen, par contre, il y a un autre type de transfert morpho-syntaxique négatif qui 
domine : 4/11 optent pour un défini (la vie), pragmatiquement peu naturel, dans le contexte donné.

En ce qui concerne la place du possessif, nous avons constaté que le système norvégien permet à la fois l'antéposition du possessif, comme dans le système français, et la postposition du possessif en combinaison avec le double marquage de définitude. Dans le texte norvégien, on trouvera des exemples comme musikken hans (sa:IRREFL musique), mannen sin (son:REFL mari), où le possessif suit le nom, et sin kone (sa:REFL femme), sin manns død (la mort de son:REFL mari), etc. avec un possessif qui précède le nom. Ces derniers emplois sont stylistiquement marqués. On pourrait alors s'attendre à ce qu'il y ait des erreurs dans les traductions des apprenants norvégiens, mais tel n'est pas le cas. Il n'y a pas la moindre erreur de ce type dans l'interlangue française des apprenants norvégiens (*musique $s a$ ). Autrement dit, la position des possessifs ne semble pas induire à des effets de transfert entre le norvégien et le français. Nous avons cependant émis l'hypothèse ailleurs (Helland 2017, 2018) que l'absence de tels cas dans notre corpus pourrait être le résultat d'un autre type de transfert. Les étudiants de nos tests apprennent le français comme leur troisième langue (L3) ayant déjà appris l'anglais comme leur L2. En anglais, les possessifs se placent, comme dans le système français, toujours devant le nom possédé : his wife - *wife his. Théoriquement parlant, on pourrait dès lors considérer l'absence absolue de possessifs postposés dans l'interlangue (L3) du français comme résultat du transfert positif de l'anglais en français (L2 $\rightarrow$ L3).

Pour résumer : sur la base de la traduction du norvégien en français, nous avons identifié un certain nombre d'effets de transfert entre le norvégien comme L1 et le français comme L2 (au sens large du terme), le transfert étant soit positif, soit négatif. Si l'on établit la distinction supplémentaire entre le français comme L3 et l'anglais comme L2, il faudra prendre en compte des effets de transfert entre la L2 de l'apprenant et le français comme L3. Pour des tests précis liés au français comme L3, il faudrait cependant y revenir dans des travaux ultérieurs. Passons maintenant à la traduction du français en norvégien.

\subsection{Traduction du français en norvégien}

Observons ce qui se passe quand l'apprenant norvégien du français passe du système possessif français au système norvégien. On part donc d'un système morphologiquement et syntaxiquement peu complexe pour viser un système plus complexe.

Quant au transfert des formes en $s^{*}$, il semble que la traduction en norvégien ne pose pas de problèmes particuliers pourvu que les conditions d'emploi d'une forme réfléchie soient claires :

(15) Il est bien rare que Nicolas Sarkozy manque un concert de sa belle Carla BruniSarkozy...

Dans un exemple comme (15), qui exige une forme réfléchie en norvégien (sa belle $C B S$ - sin:REFL vakre CBS), on trouve la forme en $s^{*}$ dans 27/33 cas dans le groupe d'Oslo et 10/13 dans le groupe de Caen. La forme réfléchie du norvégien doit être liée - en termes syntaxiques (Helland 2017, 2018) - par une expression nominale en position sujet (Nicolas Sarkozy), ce qui exclut la forme complémentaire non réfléchie (hans). Malgré ce fait, on trouve la forme non réfléchie dans 4/33 cas dans le groupe d'Oslo et 2/13 à Caen. En effet, le système de formes réfléchies et non réfléchies semble difficile à maîtriser même pour les 
étudiants norvégiens. A la troisième personne du pluriel, le norvégien oppose la forme réfléchie $s^{*}$ à la forme non réfléchie deres. Quand le possessif du français dans (16) (leurs enfants), est syntaxiquement lié par le sujet nul de la construction infinitive (= PRO), il faudrait ainsi choisir la forme réfléchie en norvégien :

(16) ... afin de permettre à son couple de $\mathrm{PRO}_{\mathrm{i}}$ retrouver rapidement leurs $\mathrm{i}_{\mathrm{i}}$ enfants.

Pour cette (dernière) phrase du texte, il y a très peu de réponses dans le groupe d'Oslo. Cependant 4/6 choisissent la forme réfléchie, 1/6 opte pour la forme (erronée) non réfléchie et 1/6 pour la forme définie sans possessif. Dans le groupe de Caen, par contre, il y a seulement $5 / 12$ de réponses avec la forme (correcte) réfléchie contre 4/12 avec la forme non réfléchie (et 3/12 avec la forme définie). Ceci montre clairement que bien des informateurs hésitent entre des formes réfléchies et non réfléchies même dans leur langue maternelle.

Cette hésitation concernant les formes en $s^{*}$ dans la production linguistique des apprenants norvégiens peut également donner des résultats surprenants :

(17) ... et chaque concert est donc l'occasion pour lui de constater que sa popularité reste intacte.

Dans (17), qui contient un groupe nominal possessif en position sujet de la subordonnée nominale, la forme réfléchie en $s^{*}$ est strictement interdite en norvégien. Cependant, dans les réponses des étudiants, on trouve la forme réfléchie (clairement erronée) dans 5/26 cas à Oslo et 2/13 cas à Caen. Ceci semble être un cas de transfert (négatif) inverse (L2 $\rightarrow$ L1) où la production linguistique de la langue maternelle de l'apprenant (L1) est directement influencée par des propriétés de la L2. Le résultat est encore une fois, chez certains informateurs, une (sur-)généralisation des formes en $\mathrm{s}^{*}$.

Pour terminer, on trouvera aussi bien dans les traductions du norvégien en français que dans celles du français en norvégien le reflet d'un autre type de transfert entre le norvégien comme L1 et le français comme L2. Nous avons montré ailleurs (Fabricius-Hansen et al, 2017, Helland 2017, 2018) que les possessifs du norvégien contrairement au français sont orientés vers le possesseur. Ainsi, les possessifs non réfléchis du norvégien (hans $N$ ( $\operatorname{son} N$ à lui) - hennes $N$ (son $N$ à elle) - deres $N$ (leur $N$ à eux), etc. varient en fonction du genre et du nombre du possesseur. En français, par contre, malgré la variation en nombre du possesseur $\left(s^{*}-\right.$ leur*), l'accord du possessif s'établit exclusivement avec le nom possédé. Dans la traduction du norvégien en français, on trouve un exemple comme musikken hans qui doit être rendu en français par sa musique sans spécification du genre du possesseur. Parmi les réponses des informateurs d'Oslo et de Caen, on trouve cependant pour un tel exemple respectivement $5 / 20$ et $4 / 14$ réponses qui optent pour un possessif masculin : *son musique. Ici, on ne peut exclure, bien sûr, le fait que les apprenants norvégiens se trompent sur le genre du nom musique (f) qui est masculin en norvégien. On parlerait alors du transfert (négatif) du trait nominal de genre entre les deux langues. Mais, il pourrait également s'agir de la volonté de la part des informateurs de préciser (incorrectement) en français le genre du possesseur comme on le fait en norvégien. Dans ce cas-là, c'est l'orientation vers le possesseur de la L1 qui est transférée dans la L2. L'exemple (18), tiré de la traduction du français en norvégien, pourrait illustrer le même type de transfert :

(18) Dès qu'il le peut, il met Carla en avant, ses talents de chanteuse, son aisance sur scène. 
Les possessifs dans (18) s'interprètent comme des formes non réfléchies, non marquées. Dans la traduction norvégienne, les répondants doivent spécifier le genre (féminin) du possesseur dans les deux cas: hennes $N\left(=s^{*} N\right.$ à elle). La grande majorité des étudiants opte pour les bonnes formes : cent pour cent de réussite dans le groupe de Caen. Cependant, dans le groupe d'Oslo 2/22 varient le genre du possesseur dans les deux cas : hennes $N$ (= ses talents à elle) et hans $N$ (= son aisance à lui). Cela montre que, du moins pour certains apprenants, les possessifs du français comme L2 sont orientés vers le possesseur sur le modèle des possessifs norvégiens, conséquence supplémentaire du transfert entre le norvégien L1 et le français L2.

\section{Conclusion}

Nous avons pris pour point de départ la notion de transfert dans la littérature linguistique consacrée à l'acquisition des langues étrangères. Si le transfert s'applique traditionnellement aux domaines phonétiques-phonologiques et lexicaux, notre intention a été de justifier l'existence d'effets de transfert également au niveau de la morphologie et de la syntaxe. Pour appuyer notre argumentation, nous avons comparé les systèmes possessifs du norvégien comme L1 et du français comme L2, éventuellement L3. Les possessifs illustrent de façon exemplaire les influences bi-directionnelles entre le norvégien et le français dans le processus acquisitionnel : il peut s'agir de transfert entre le norvégien comme L1 et le français comme L2 (L1 $\rightarrow$ L2) ou inversement entre le français comme L2 et le norvégien comme L1 $(\mathrm{L} 2 \rightarrow \mathrm{L} 1)$. Le transfert peut s'étendre également au français comme L3 entraînant (possiblement) des effets de transfert entre l'anglais comme L2 et le français comme L3 (L2 $\rightarrow$ L3). Les exemples sur lesquels nous nous sommes appuyés sont tirés de tests de traduction effectués avec des apprenants universitaires norvégiens étudiant le français à l'Université d'Oslo ou dans un contexte d'immersion (à l'Université de Caen).

\section{Bibliographie}

Alessandro G. Benati \& Tanja Angelovska, 2016. Second Lanugage Acquisition. London: Bloomsbury.

Eide, Kristin Melum (éd.), 2015. Norsk andrespråkssyntaks. Trondheim: Novus.

Ellis, Rod, 2008. The Study of Second Language Acquisition. Oxford: Oxford University Press.

Fabricius-Hansen, Cathrine, Helland, Hans Petter, Pitz, Anneliese, 2017. An L2 perspective on possessives: contrasts and their possible consequences. C. Fabricius-Hansen et al. (éd.) Possessives in L2 and translation: basic principles and empirical findings. OSLa Oslo Studies in Language. Volume 9, numéro 2. 3-39.

Helland, Hans Petter, 2017. An empirical L2 perspective on possessives: French/Norwegian. C. Fabricius-Hansen, et al. (éd.) Possessives in L2 and translation: basic principles and empirical findings. OSLa Oslo Studies in Language. Volume 9, numéro 2. 75-104.

Helland, Hans Petter, 2018. Complexité et fréquence: L'acquisition des structures possessives. Synergies Pais Scandinaves, 37-50. 
Jarvis, Scott. Transfer: An Overview with an Expanded Scope. A. Golden et al. (éd.) Crosslinguistic Influence and Distinctive Patterns of Language. Bristol:Multilingual matters. 27-45.

Jarvis, Scott \& Aneta Pavlenko, 2008. Crosslinguistic Influence in Language and Cognition. London \& New York: Routledge.

Lorentzen, Lise Richter, 2010. Dictionnaire des faux amis norvégiens-français. Oslo: Unipub.

Odlin, Terence, 2003. Cross-linguistic influence. C.J. Doughty \& M.H. Long (éd.)The Handbook of Second Language Acquisition. London : Blackwell. 436-86.

Odlin, Terence \& Liming Yu, 2016. Introduction. L. Yu et al. (éd.) New Perspectives on Transfer in Second Language Learning. Bristol : Multilingual Matters. 1-16.

Woldsnes, Anne Kathrine, 2013. La possession inaliénable - une analyse contrastive. Oslo: University of Oslo. 